\title{
Ergodic Properties of an Infinite System of Particles Moving Independently in a Periodic Field $\star$
}

\author{
Sheldon Goldstein \\ Institute for Advanced Study, Princeton, N.J., USA \\ Joel L. Lebowitz \\ Belfer Graduate School of Science, Yeshiva University, New York, N. Y., USA
}

Received January 5, 1974

\begin{abstract}
We investigate the ergodic properties of a general class of infinite systems of independent particles which undergo nontrivial "collisions" with an external field, e.g. fixed convex barriers (the Lorentz gas). We relate the ergodic properties of these systems to the ergodic properties for a single particle moving in a finite box (with periodic boundary conditions) with the same dynamics. We prove that when the one particle system is mixing or a $K$-system for a sequence of boxes approaching infinity so is the infinite particle system with an equilibrium measure obtained as a Poisson construction over the one particle phase space.
\end{abstract}

\section{Introduction}

The ergodic properties of some "interesting" finite Hamiltonian systems are known in considerable detail $[1,2]$. Very little is known, however, about the ergodic properties of nontrivial systems with an infinite number of degrees of freedom. These are of great interest for statistical mechanics. Sinai [3, 4], De Pazzis [5] and more recently, Aizenman, Goldstein, and Lebowitz [6] have investigated the ergodic properties of an infinite ideal gas in an arbitrary number of dimensions and of an infinite system of hard rods moving in one dimension while Lanford and Lebowitz [7] have considered the infinite harmonic system. While these systems have very good ergodic properties $(K$ or Bernoulli systems) the physical interpretation of this result is that "local disturbances stream off to infinity where they are no longer visible" [8]. Thus these results shed little light on the "local approach to equilibrium" for macroscopic systems.

We investigate here the ergodic properties of some infinite systems with non-trivial "collisions", i.e., the transformation which occurs during a collision possesses itself good mixing properties. Except for these col-

* Research sponsored in part by the Air Force Office of Scientific Research Grant No. 73-2430 A and The National Science Foundation Grant No. GP-16147 A, No. 1. 
lisions the particles move freely. The main result of this paper is contained in Theorem 7.1. There the ergodic properties of an infinite system of non-interacting particles moving in a periodic field (e.g., fixed barriers with which they undergo collisions) is related to the ergodic properties of a single particle moving in a finite box (with periodic boundary conditions) with the same dynamics.

It seems reasonable to expect that the ergodic properties of our systems will be at least as good as those of the "trivial" systems mentioned earlier. We must be cautious, however, since the physical explanation of the ergodic properties of those systems may not be valid here. It will be seen, in fact, that the underlying mathematical structures (partitions) which determine the ergodic properties of the systems are of a very different nature from those obtained by Sinai for the infinite ideal gas.

\section{General Description of a One-Dimensional Model System}

We observe that insofar as we will be concerned with systems of noninteracting particles, we expect that the measure spaces of our infinite systems can be identified with "Poisson distributions" of points in the one-particle measure spaces, with the infinite system dynamics induced by that of the one-particle systems.

To develop the general theory of such systems we begin with the investigation of the ergodic properties of an infinite system of non-interacting particles moving freely in one dimension except for "collisions". A periodic array of barriers is the agency responsible for the collisions; when a particle reaches a barrier, it may be either reflected or transmitted. Since we wish to study a dynamical system, we attach to each particle internal parameters whose sole function is to determine which of those two possibilities occurs. In turn the sole effect of the spatial variables (position and velocity) upon the internal dynamics will be assumed to be the determination of the times at which the internal parameters undergo a transformation. We choose the convention that the transformation occurs immediately after a particle leaves a barrier.

We choose as our internal dynamical system one which, though among the simplest of dynamical systems, has ergodic properties of the strongest kind (Bernoulli): the Bernoulli shift on an alphabet of 2 letters each with weight $\frac{1}{2}, B\left(\frac{1}{2}, \frac{1}{2}\right)$, which is equivalent to the baker's transformation [9]. It is also natural from the standpoint of the theory of Bernoulli shifts to require that the spatial dependence upon the internal space should be measurable with respect to the partition which determines the entire ergodic structure of the internal dynamics, the independent generator [10]. This is the 2-element partition of the 
baker's square into a left side and a right side (of the same area). The dynamics can therefore be described as follows: a particle moves freely until it comes to a barrier; if its internal parameters lie in the left side of the baker's square the particle is reflected, otherwise it is transmitted. In either case the internal variables undergo a baker's transformation, and the particle moves on freely until it reaches another barrier.

Since the particles are non-interacting nothing would be gained from a purely dynamical point of view by our considering an infinite system of particles; we are considering an infinite system because we are interested in ergodic properties. Thus we must specify an invariant probability measure on the phase space. Such a measure should be natural from the standpoint of statistical mechanics, e.g., a limit of grand canonical measures on finite systems [11]. The simplest measure satisfying these requirements may be described as follows: (a) the (unlabelled) particles are distributed along the line with a Poisson distribution of density $\varrho$, (b) the internal and velocity spaces associated with a particle at a given position are independent of the configuration (positions) of the particles, of the spaces associated with other particles, and of each other.

We note that whereas it is only in the infinite case that the ideal gas becomes ergodically interesting, our system, since it has a nontrivial dynamics, is ergodically interesting even for a single particle. We shall therefore investigate this problem first.

\section{Ergodic Properties of One-Particle System}

Let the barriers be situated at integral positions. Choose the unit of time so that the absolute value of the velocity of the particle is unity. (The speed of the particle is a constant of the motion.) The only modification of the description in the preceding section is that we now take for our external space $\mathbb{R} / n \mathbb{Z}$, the real line modulo some integer $n$, instead of $\mathbb{R}$, i.e. the particle moves on a circle $L$ of length $n$. This is necessary because we wish to have a normalized spatially homogeneous invariant measure.

We thus have the following dynamical system $\tau=\left(X, \Sigma, \mu, S_{t}\right)$ : The phase space $X=\mathbb{R} / n \mathbb{Z} \otimes\{1,-1\} \otimes B$, where $B$ is the baker's square. The $\Sigma$-algebra $\Sigma=\Sigma_{L} \otimes P\{1,-1\} \otimes \Sigma_{B}$ where $\Sigma_{L}$ is the $\Sigma$-algebra of Lebesgue sets of the real line modulo $n, P\{1,-1\}$ is the power set of $\{1,-1\}$ (regarded as a $\Sigma$-algebra), and $\Sigma_{B}$ is the $\Sigma$-algebra of Lebesgue sets of the baker's square. The measure $\mu=\mu_{L} \otimes \mu_{2} \otimes \mu_{B}$, where $\mu_{L}$ is the normalized Lebesgue measure on $\Sigma_{L}, \mu_{2}$ assigns mass $\frac{1}{2}$ to the points of $\{1,-1\}$ and $\mu_{B}$ is the normalized Lebesgue measure on the baker's 
square. $S_{t}$ is a measurable flow on $X$ such that for $t<1$ we have

$$
S_{t}(x, v, \xi)=\left\{\begin{array}{l}
(x+v t, v, \xi), \quad \text { if } \mathbb{Z} \cap[(x, x+v t) \cup(x+v t, x)]=\emptyset \\
\left(m+\xi_{0} v(t-|m-x|), \xi_{0} v, T \xi\right), \\
\text { if } \mathbb{Z} \cap[(x, x+v t) \cup(x+v t, x)]=m .
\end{array}\right.
$$

Here $x \in \mathbb{R} / n \mathbb{Z}, v \in\{1,-1\}, \xi \in B, T$ is the baker's transformation, and $\xi_{k}= \pm 1$ is the $k$ th coordinate of the Bernoulli representation of $\xi$ [9].

One easily checks that the above does in fact describe a dynamical system, and in particular that $\mu$ is invariant under $S_{t}$. However, it is not difficult to see that this dynamical system is not mixing; in fact, $S_{1}$ is not even ergodic. If $A=\bigcup_{k=0}^{n-1}\left(k+\frac{1}{4}, k+\frac{3}{4}\right)$ then $A \otimes\{1,-1\} \otimes B$ is a subset of $X$, invariant under $S_{1}$, with measure $\frac{1}{2}$. More generally, all periodic functions of $\mathbb{R} / n \mathbb{Z}$ symmetric about the point $x=\frac{1}{2}$ are invariant under $S_{1}$.

The failure of $\tau$ to possess strong mixing properties is not very surprising; the breakdown occurs in precisely that "part" of $\tau$ which is in no way affected by the good mixing properties which we built into the collisions. To be more precise, let us define a bijection $\alpha$ from $X$ to $X^{\prime}=\{0, \ldots, n-1\} \otimes[0,1) \otimes\{1,-1\} \otimes B$ as follows: Let $m$ be the position of the last barrier with which the particle in the dynamical state $u \in X$ has collided. Let $t_{0}<0$ be the time when the last collision has taken place; $t_{0}>-1$. Then $\alpha(x, v, \xi)=\left(m,\left|t_{0}\right|, v, \xi\right)$. Thus $\alpha$, regarded as a mapping defined on the configurational part of $X$, can be thought of as a transformation from the position coordinate $x$ to coordinates $\left(m_{0}, \delta\right)$ which describe the location of the barrier from which the particle is departing and the distance of the particle from this barrier, respectively. $\alpha$ determines, in an obvious manner, a dynamical system $\tau^{\prime}$ which is isomorphic to $\tau$. Letting $S_{t}^{\prime}$ be the image of $S_{t}$ under $\alpha$, we have

$$
S_{t}^{\prime}(m, \delta, v, \xi)=\left\{\begin{aligned}
(m, \delta+t, v, \xi) & \text { if } 0 \leqq t<1-\delta \\
\left(m+v,(\delta+t) \bmod 1, \xi_{0} v, T \xi\right) & \text { if } 1 \geqq t>1-\delta
\end{aligned}\right.
$$

We thus see that $S_{t}^{\prime}$ acts in a trivial way upon $[0,1)$ (the second term in the product defining $X^{\prime}$ ). Indeed $\tau^{\prime}$ can be factored into a skew product with a rotation for its first component:

$$
\begin{aligned}
S_{t}^{\prime}(\delta, \omega) & \left.=(\delta+t) \bmod 1, \varphi_{t}^{\delta} \omega\right), \quad \delta \in[0,1), \\
\omega & \in\{0, \ldots, n-1\} \otimes\{-1,1\} \otimes B
\end{aligned}
$$

in an obvious manner. 
Note that although $\varphi_{t}^{\delta}$ does not form a one parameter group, its value changes only when $t=k-\delta, k \in \mathbb{Z}$, and $\varphi_{k}^{\delta}=\left(\varphi_{1}^{\delta}\right)^{k}=\left(\varphi_{1}\right)^{k}$, since $\varphi_{1}^{\delta}$ is independent of $\delta$. Thus $\tau$ "factors" into the product of a rotation and an essentially discrete (space and time) dynamical system $\bar{\tau}^{\prime}$ in which all of the ergodic activity occurs. We investigate such a system in the next section.

\section{Discrete One Particle System}

The discrete dynamical system $\bar{\tau}^{\prime}$ can be described as follows:

$$
\begin{gathered}
\bar{\tau}^{\prime}=\left(\bar{X}^{\prime}, \bar{\Sigma}^{\prime}, \bar{\mu}^{\prime}, \overline{S^{\prime}}\right) \\
\bar{X}^{\prime}=\mathbb{Z}_{n} \otimes\{-1,1\} \otimes B, \quad\left(\mathbb{Z}_{n} \text { is the set of integers } \bmod n\right) \\
\bar{S}^{\prime}(m, v, \xi)=\left(m+v, \xi_{0} v, T \xi\right)
\end{gathered}
$$

where $v= \pm 1$ and $\overline{\Sigma^{\prime}}$ and $\vec{\mu}$ are obvious.

Since we are now dealing with a discrete system, the velocities are somewhat unnatural. Therefore, instead of investigating $\bar{\tau}^{\prime}$ we will investigate an apparently simpler system $\bar{\tau}$ which has the same ergodic properties as $\bar{\tau}^{\prime} . \bar{\tau}$ is obtained from $\bar{\tau}^{\prime}$ by dropping the velocity part of phase space and making the appropriate modification of the dynamics; $\bar{\tau}$ is, in fact, isomorphic to $\bar{\tau}^{\prime}$.

We let $\bar{\tau}(n)=(\bar{X}, \bar{\Sigma}, \bar{\mu}, \bar{S})$ where $\bar{X}=B \otimes \mathbb{Z}_{n}, \bar{\Sigma}$ and $\bar{\mu}$ are the corresponding $\Sigma$-algebra and measure, and $\bar{S} x=\bar{S}(\xi, k)=\left(T \xi, k+\xi_{0}\right) . \bar{\tau}(n)$ is thus a skew product of a Bernoulli shift with a rotation on the integers $\bmod n$. The dependence of the rotation on $\xi$ is measurable with respect to an independent generator of $B[10]$. It is known $[10,12]$ that such a system is Bernoulli if it is mixing. We will here prove as a special case the following:

Theorem 4.1. $\bar{\tau}(n)$ is Bernoulli if and only if $n$ is odd. (For $n$ even $\bar{\tau}(n)$ fails to be mixing.)

Proof. Let $M_{n}$ be the Markov shift on $\mathbb{Z}_{n}$ with transition probabilities $\pi_{j k}=\frac{1}{2}\left(\delta_{j, k+1}+\delta_{j, k-1}\right)$ and stationary distribution $p_{k}=1 / n$ (random walk). Since a mixing Markov shift is Bernoulli [10], the theorem follows from 2 lemmas:

Lemma 4.2. $\bar{\tau}(n)$ is isomorphic to $M_{n}$.

Lemma 4.3. $M_{n}$ is mixing if and only if $n$ is odd.

Proof of Lemma 4.3. A Markov shift is mixing if and only if the $m$ th order transition probabilities $\pi_{j k}^{m}$ approach (in the limit $m \rightarrow \infty$ ) the stationary distribution $p_{k}[13]$. 
For a Markov chain on a finite state space this condition holds if the chain is irreducible and aperiodic [14]. (A chain is irreducible if every state has a nonvanishing probability of being reached from any other state. A chain is aperiodic if every state has Period 1. If $v$ is the largest integer such that $\pi_{j j}^{n}$ is nonvanishing only for $n$ an integral multiple of $v$, the state $j$ is said to have period $v$.)

It is clear that $M_{n}$ is irreducible for all $n$. For $n$ even all states have Period 2, since the states can be partitioned into an "even" class and an "odd" class in such a way that (one-step) transitions always involve a change in class. For all $n$ we have $\pi_{j j}^{2} \geqq 0$, so $v \leqq 2$. Since by jumping to the right on each transition the system will eventually return to its initial state, we have $v=1$ for $n$ odd. Thus for $n$ odd $M_{n}$ is aperiodic and the lemma is established.

Proof of Lemma 4.2. One easily checks that

$$
P_{n}=\left\{C_{k}^{[n]}\right\}=\left\{\left\{(\xi, j) \mid \xi \in B, j=k \in \mathbb{Z}_{n}\right\}\right\}
$$

is a Markov generator [10] for $\bar{S}$, having the same conditional probabilities as $M_{n}$. (The isomorphism $(\bmod 0), \alpha$, determined by $P_{n}$ is easily seen to map every point $x \in \bar{X}$ into its trajectory $\left\{\eta_{k}\right\} \in \mathbb{Z}_{n}^{\mathbb{Z}}$. Equipped with the measure induced by $\alpha, \mathbb{Z}_{n}^{\mathbb{Z}}$ becomes the measure space of $M_{n}$ and the image of $\bar{S}$ under $\alpha$ is clearly the shift on trajectories.)

In the next section we will have occasion to use a general criterion for determining whether a countable family $\Gamma$ of measurable subsets of a Lebesgue space $[15](X, \Sigma, \mu)$ generates $\Sigma$. A necessary and sufficient condition for $\Gamma$ to generate $(\bmod 0)$ is that the decomposition $\zeta(\Gamma)$ determined by $\Gamma$ be the decomposition of $X$ into points $(\bmod 0)$; i.e., that there exist a set $A$ of full measure such that for any $x, y \in A$ there exists $\Gamma_{n} \in \Gamma$ for which $x \in \Gamma_{n}, y \notin \Gamma_{n}$ or $y \in \Gamma_{n}, x \notin \Gamma_{n}$ [15]. For the case that $\Gamma$ is generated from a (finite) partition $P$ by the transformation $T$ this condition reduces to the requirement that the mapping from points to trajectories determined by $(P, T)$ be injective (mod 0$)$. It is trivial to check that for the system $\bar{\tau}(n),\left(P_{n}, \bar{S}\right)$ satisfies this condition (everywhere).

\section{Ergodic Properties of Infinite Discrete System}

We have now descended as far as we will go in the direction of simplifification, and we will now begin an ascent to the general systems with which we are primarily concerned. We investigate a system $\bar{\tau}_{\infty}$ which is essentially the thermodynamic limit of the model of the previous section. (Since the particles are non-interacting in all of the models which we consider, there is nothing to be gained by considering a system with several particles.) We expect the infinite system to have "strong" ergodic 
properties, having found finite systems for which this is the case and remembering that the thermodynamic limits of some trivial systems (i.e. the ideal gas) possess these properties.

The system $\bar{\tau}_{\infty}(\varrho)=\left(\bar{X}_{\infty}, \bar{\Sigma}_{\infty}, \bar{\mu}_{\infty}(\varrho), \bar{S}_{\infty}\right)$ can be most easily described by means of a Poisson construction. In general, given $(X, \Sigma, \mu, T)$, an automorphism of a totally $\sigma$-finite non atomic measure space, we may obtain a probability space $\left(X_{\infty}, \Sigma_{\infty}, \mu_{\infty}\right)$ with automorphism $T_{\infty}$, the Poisson system built over $(X, \Sigma, \mu, T)$, by means of a Poisson construction: We let $X_{\infty}$ denote the set of countable subsets of $X$. For any $A \in \Sigma$ we define a function $N(A)$ by

$$
[N(A)](x)=\#(A \cap X),
$$

for $x \in X_{\infty}$ and where "\#" denotes "cardinality of". We then let $\Sigma_{\infty}$ be the $\Sigma$-algebra generated by all the $N(A)^{1}$, and we let $\mu_{\infty}$ be the measure for which each $N(A)$ has a Poisson distribution with mean $\mu(A)$ (i.e.,

$$
\left.\mu_{\infty}\left\{x \in X_{\infty} \mid[N(A)](x)=m\right\}=\exp (-\mu(A)) \mu(A)^{m} / m !\right)
$$

and for which disjoint regions of $X$ support independent $\Sigma$-algebras. ( $\Sigma_{A}$, the $\Sigma$-algebra supported by $A$, is generated by the random variables $N(B)$ with $B \subset A)$. Finally, for $x \in X_{\infty}$ we let

$$
T_{\infty} x=T x,
$$

where on the right side of the equation we explicitly regard $x$ as a subset of $X$. That $T_{\infty}$ preserves $\mu_{\infty}$ follows easily from the $\mu$-preserving nature of $T$, as will be illustrated in the proof of Theorem 5.1.

We now identify $\bar{\tau}_{\infty}(\varrho)$ with the Poisson system built over the generalized baker's transformation $\left(\bar{B}_{\infty}, \Sigma_{\bar{B}_{\infty}}, \varrho \mu_{\bar{B}_{\infty}}, \bar{T}_{\infty}\right)^{2}$, where

$$
\left(\bar{B}_{\infty}, \Sigma_{\bar{B}_{\infty}}, \mu_{\bar{B}_{\infty}}\right)=\bigoplus_{i=\infty}^{\infty} \delta_{i}
$$

with $\delta_{i}=\left(B, \Sigma_{B}, \mu_{B}\right)$, the measure space of the baker's transformation, for all $i$. Identifying $\bar{B}_{\infty}$ with $B \otimes \mathbb{Z}$, etc., we will write $B_{i}$ for $B \otimes i$, and define $\bar{T}_{\infty}$ by $\bar{T}_{\infty}(\xi, m)=\left(T \xi, m+\xi_{0}\right) . \bar{T}_{\infty}$ has a simple geometric representation: Recall that the baker's transformation can be described geometrically as a two step process:

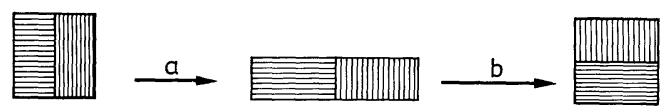

Now if we perform the baker's transformation independently on a doubly infinite array of baker's squares and follow it with the simultaneous

\footnotetext{
${ }^{1}$ Any set $A$ to which we shall henceforth refer is to be understood as measurable $(\in \Sigma)$.

${ }^{2}$ We will usually delete the reference to $\varrho$ in $\bar{\tau}_{\infty}(\varrho)$.
} 
translation of the top half of each resulting square one unit to the right and the bottom half one unit to the left, we obtain $\bar{T}_{\infty}$. We thus have the following "picture" of $\bar{T}_{\infty}$ :
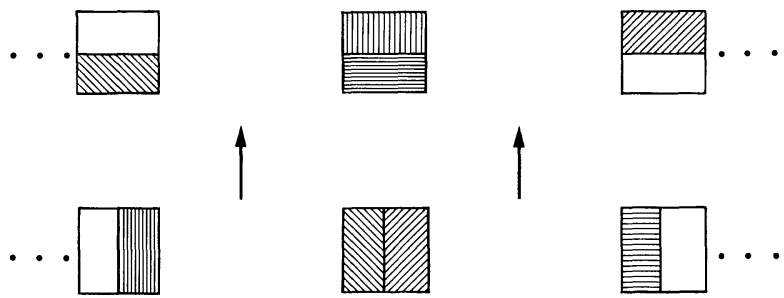

This auxiliary system is, of course, simply a one particle component of $\bar{\tau}_{\infty}$, i.e., the infinite volume $(n \rightarrow \infty)$ limit of the $\bar{\tau}(n)^{3}$.

We shall now show that the natural requirements of a) independent probabilities at different sites (since there are no interactions) and b) the homogeneity of the baker's square, i.e., if $A, D \subset B_{i}$ with $\mu_{B}(A)=\mu_{B}(D)$, then $N(A)$ and $N(D)$ are identically distributed, make $\bar{\mu}_{\infty}$ the only "reasonable" invariant probability measure for this problem. While this is in agreement with our intuition it is not immediately obvious that the number of particles at a given site must have a Poisson distribution. That this is so is proven in the following theorem.

Theorem 5.1. $\bar{\mu}_{\infty}$ is the unique $\bar{\tau}_{\infty}$-invariant probability measure on $\bar{\Sigma}_{\infty}$ for which we have:

a) The $\Sigma_{B_{i}}, i=0,1,-1 \ldots$, form an independent sequence of $\Sigma$ algebras.

b) The distribution of $N(A), A \subset B_{i}, i \in \mathbb{Z}$, is determined by $\mu_{\bar{B}_{\infty}}(A)$ (and i) and satisfies $\langle N(A)\rangle=\int d \bar{\mu}_{\infty}(x)[N(A)](x)<\infty$.

Proof. We note that

$$
\left[U^{-1} N(D)\right](x) \equiv[N(D)]\left(\bar{S}_{\infty}^{-1} x\right)=\left[N\left(\bar{T}_{\infty} D\right)\right](x)
$$

and that

$$
\begin{aligned}
\bar{S}_{\infty}\left(\left\{x \in \bar{X}_{\infty} \mid[N(D)](x)=m\right)=\left\{\bar{S}_{\infty} x \mid[N(D)](x)=m\right\}\right. \\
\quad=\left\{x \mid[N(D)]\left(\bar{S}_{\infty}^{-1} x\right)=m\right\}=\left\{x \mid\left[N\left(\bar{T}_{\infty} D\right)\right](x)=m\right\} .
\end{aligned}
$$

It now follows from the area $\left(\mu_{\bar{B}_{\infty}}\right)$ preserving property of $\bar{T}_{\infty}$ that any measure for which the joint distribution of any finite sequence of random variables of the form $\left\{N\left(D_{i}\right)\right\}$, where $\left\{D_{i}\right\}, i \in Z$, is a sequence of pairwise disjoint subsets of $\bar{B}_{\infty}$, depends only on the sequence of areas $\left\{\mu_{\bar{B}_{\infty}}\left(D_{i}\right)\right\}$ is

${ }^{3}$ One easily verifies that $\bar{\tau}_{\infty}$ is equivalent to the discrete analogue of the system described in Section 2. 
invariant under $\bar{S}_{\infty} \cdot \bar{\mu}_{\infty}$ is thus invariant (since $\{N(D)\}$ is Poisson with constant density).

To prove uniqueness it is sufficient to show that $\Sigma_{A}$ is independent of $\Sigma_{C}$ when $A \cap C=\emptyset$. We can assume that $A$ and $C$ are both subsets of $B_{0}$. If $A_{0}$ and $C_{0}$ are distinct atoms of

$$
\bigvee_{k=-n}^{n} T^{k} P\left(P=\left\{P_{i}\right\}, P_{i}=\left\{\xi \in B \mid \xi_{0}=i\right\}\right),
$$

there exists $j \in[-n, n+1]$ for which $\bar{T}_{\infty}^{j} A_{0} \subset B_{l}$ and $\bar{T}_{\infty}^{j} C_{0} \subset B_{m}, l \neq m$. Thus $\Sigma_{\bar{T}_{\infty}^{j} A_{0}}=\bar{S}_{\infty}^{j} \Sigma_{A_{0}}$ is independent of $\bar{S}_{\infty}^{j} \Sigma_{C_{0}}=\Sigma_{\bar{T}_{\infty}^{j} C_{0}}$ so that, by invariance, $\Sigma_{A_{0}}$ is independent of $\Sigma_{C_{0}}$. By an induction on $n$ one verifies that for any $N$, and for $A^{\prime}$ and $B^{\prime}$ disjoint unions of atoms of $\bigvee_{-N}^{N} T^{j} P$, $\Sigma_{A^{\prime}}$ is independent of $\Sigma_{B^{\prime}}$. Because $\langle N(D)\rangle=\langle N(E)\rangle$ for $\mu_{\bar{B}_{\infty}}(D)=\mu_{\bar{B}_{\infty}}(E)$, $N(D)=0$, a.e., if $\mu_{\bar{B}_{\infty}}(D)=0$. Thus, since $P$ is a generator for $T, \Sigma_{A}$ must be independent of $\Sigma_{B}$ for $A \cap B=\emptyset$. Since $\left\langle N\left(B_{i}\right)\right\rangle$ clearly equals $\left\langle N\left(B_{j}\right)\right\rangle$, we are done.

Using methods similar to those used above, we prove the following:

Theorem 5.2. $\bar{\tau}_{\infty}$ is mixing.

Proof. Let $\alpha=\left\{\alpha_{j}\right\}, j \in J$ and $\beta=\left\{\beta_{k}\right\}, k \in K$, be finite families of disjoint subsets of $\bar{B}_{\infty}$ such that $\bigcup_{j \in J} \alpha_{j}$ and $\bigcup_{k \in K} \beta_{k}$ are contained in $\bigcup_{i=-N}^{N} B_{i}$ for some $N$. Let any set $\alpha_{k} \in \alpha$ be an atom of $\bigvee_{j=-M}^{M} T^{j} P^{i}$ for some $M$ and $-N \leqq i<N$, where $P^{i}$ is the partition of $B_{i}$ corresponding to the partition $P$ of $B$. Let

$$
X_{\alpha}^{\left\{n_{j}\right\}}=\left\{x \in \bar{X}_{\infty} \mid N\left(\alpha_{j}\right)=n_{j} \text { for all } j \in J\right\}
$$

and

$$
X_{\beta}^{\left\{m_{k}\right\}}=\left\{x \in \bar{X}_{\infty} \mid N\left(\beta_{k}\right)=m_{k} \text { for all } k \in K\right\} .
$$

We have $\bar{S}_{\infty} X_{\alpha}^{\left\{n_{j}\right\}}=X_{T_{\infty} \alpha}^{\left\{n_{j}\right\}}$. Also $\bar{T}_{\infty}^{M} \alpha$ is a family which is independent of the "future". Thus $\bar{T}_{\infty}^{M+m}, m=1,2 \ldots$ induces a random walk on a point uniformly distributed over an element of $\alpha$. We can, therefore, use the central limit theorem to find an $\bar{N}$ so large that for $\bar{N} \geqq \bar{N}$

$$
\mu_{\bar{B}_{\infty}}\left(\bigcup_{j} \bar{T}_{\infty}^{\bar{N}} \alpha_{j} \cap \bigcup_{i=-N}^{N} B_{i}\right)<\varepsilon .
$$

We can now use the independence of $N(A)$ and $N(C)$ for $A \cap C=\emptyset$ to conclude that

$$
\begin{aligned}
\lim _{n \rightarrow \infty} \bar{\mu}_{\infty}\left(\bar{S}_{\infty}^{n} X_{\alpha}^{\left\{n_{3}\right\}} \cap X_{\beta}^{\left\{m_{k}\right\}}\right) & \\
= & \lim _{n \rightarrow \infty} \bar{\mu}_{\infty}\left(X_{\bar{T}_{\infty} \alpha}^{\left\{n_{j}\right\}} \cap X_{\beta}^{\left\{m_{k}\right\}}\right)=\bar{\mu}_{\infty}\left(X_{\alpha}^{\left\{n_{j}\right\}}\right) \bar{\mu}_{\infty}\left(X_{\beta}^{\left\{m_{k}\right\}}\right) .
\end{aligned}
$$


Since finite unions of sets of the form $X_{\alpha}^{\left\{n_{j}\right\}}$ are dense in $\bar{\Sigma}_{\infty}$, we conclude that $\bar{\tau}_{\infty}$ is mixing.

We will now show that $\bar{\tau}_{\infty}$ is a $K$-system. We first review the definition. A continuous Lebesgue space $(X, \Sigma, \mu)$ equipped with an invertible measure preserving transformation $S$ is said to be a $K$-system if there exists a measurable partition $\zeta_{0}$, called a $K$-partition, such that [15-18]

1) $S^{n} \zeta_{0}=\zeta_{n} \geqq \zeta_{0}(\bmod 0)$ for $n \geqq 0$;

2) $\bigvee_{n} \zeta_{n}=\varepsilon(\bmod 0)$, where $\varepsilon$ is the partition of $X$ into its elements;

3) $\bigwedge_{n} \zeta_{n}=v(\bmod 0)$, where $v$ is the trivial partition of $X$ whose sole element is $X$ itself.

If $\left\{S^{t}\right\}$ is a measurable flow on $(X, \Sigma, \mu)$ and if in the above definition we replace " $n$ " by " $t$ ", we obtain the definition of a $K$-flow.

We have already shown that the single particle system $\bar{\tau}(n)$ is Bernoulli, which implies that it is a $K$-system, for $n$ odd. Let $\Sigma_{n}^{\prime}$ be the $\Sigma$-algebra generated by the $\bar{S}^{k} P_{n}, k \leqq 0$, where $P_{n}$ was defined in Section 4 , and let $\zeta\left(\Sigma_{n}^{\prime}\right)$ be the partition determined by the family of sets of the form $\bar{S}^{k} P_{n, i}$, $k \leqq 0, P_{n, i} \in P_{n}[15]$. It is easy to see that $\zeta\left(\Sigma_{n}^{\prime}\right)$ satisfies 1$\left.)-3\right)$. 1) is trivial, 2 ) is equivalent to the fact that the $\bar{S}^{k} P_{n}, k \in \mathbb{Z}$ generate (see final paragraph of Section 4), and 3) follows from the fact that for $n$ odd the transition probabilities approach the values of the stationary distribution. (That the partitions which we encounter are measurable and that the spaces are Lebesgue are easily verifiable in each case [19].) $\zeta\left(\Sigma_{n}^{\prime}\right)$ can be described as that partition for which $x \sim x^{\prime}$, i.e., $x=(\xi, m)$ and $x^{\prime}=\left(\xi^{\prime}, m^{\prime}\right)$ belong to the same element of $\zeta\left(\Sigma_{n}^{\prime}\right)$, when $m=m^{\prime}$ and $\xi_{j}=\xi_{j}^{\prime}$ for $j \geqq 0$.

We now introduce some notation for partitions of $\bar{X}_{\infty}$. Let $\bar{\gamma}$ be a partition of $\bar{B}_{\infty}$. We denote by $\zeta[\bar{\gamma}]$ the partition of $\bar{X}_{\infty}$ generated by functions of the form $N(D), D \in \Sigma(\bar{\gamma}) \subset \Sigma_{\bar{B}_{\infty}}[15,16]$. Let $\hat{\gamma}$ denote the partition for which $B_{i} \in \Sigma(\hat{\gamma})$ for all $i$ and which when restricted to each $B_{i}$ is "identical" to $\gamma$ (a partition of $B$ ). We write $\zeta[\gamma]$ for $\zeta[\hat{\gamma}]$. We denote by $\gamma_{0}$ the partition of $B$ into vertical line segments, i.e., $\xi \sim \xi^{\prime}$ when $\xi_{j}=\xi_{j}^{\prime}$ for $j \geqq 0$. We recall that $\gamma_{0}$ is a $K$-partition for $\left(B, \Sigma_{B}, \mu_{B}, T\right)$. We shall now show that $\zeta\left[\gamma_{0}\right]$ is a $K$-partition for $\bar{\tau}_{\infty}$.

Theorem 5.3. $\bar{\tau}_{\infty}$ is a K-system.

Proof. We will show that $\zeta\left[\gamma_{0}\right]$ satisfies 1$\left.)-3\right)$. We observe that

a) $\bar{S}_{\infty}^{n} \zeta[\gamma]=\zeta\left[\bar{T}_{\infty}^{n} \gamma\right], n \in \mathbb{Z}$ and

b) $\bar{S}_{\infty}^{n} \zeta[\gamma]=\zeta\left[T^{n} \gamma\right], n \in \mathbb{Z}^{+}, \gamma$ a partition of $B$, and $\gamma \geqq P$.

1) Is an immediate consequence of $b$ ) and the corresponding property of $\gamma_{0}$. Similarly, 2) follows from $\left.b\right)$ and the fact that $\bigvee_{n} T^{n} \gamma_{0}=\varepsilon_{B}(\bmod 0)$ : for $x \neq y \in \bar{X}_{\infty}$, there exists an $N$ and a set $A$ such that $[N(A)](x)$ 
$\neq[N(A)](y), A \in \bigvee_{j=-N}^{N} T^{j} P^{i}$ for some $i \in \mathbb{Z}$. Thus $x$ is separated from $y$ by $\zeta\left[T^{N} \gamma_{0}\right]$ and hence by $\bigvee_{n} \bar{S}_{\infty}^{n} \zeta\left[\gamma_{0}\right]$. Hence $\bigvee_{n} \bar{S}_{\infty}^{n} \zeta\left[\gamma_{0}\right]=\varepsilon\left(=\bigvee_{n}\right.$ $\left.\cdot \zeta\left[T^{n} \gamma_{0}\right]=\zeta\left[\bigvee_{n} T^{n} \gamma_{0}\right]=\zeta\left[\varepsilon_{B}\right]\right)(\bmod 0)$

We now give a (somewhat) heuristic argument for 3). Let $\sigma^{n}=\Sigma\left(\bar{S}_{\infty}^{-n} \zeta\left[\gamma_{0}\right]\right)$ and let $\sigma=\bigcap_{n} \sigma^{n}$. To establish 3) we must show that if $A \in \sigma$ we have $\bar{\mu}_{\infty}(A)=0$ or $\bar{\mu}_{\infty}(A)=1$. Let $F_{n}$ be the $\Sigma$-algebra generated by $\left\{N(D): D \subset \bigcup_{i=-n}^{n} B_{i}\right\}$. Let $F=\bigcup_{n>0} F_{n}$. We would like to show that if $C \in F$ and if $A \in \sigma$ then $\bar{\mu}_{\infty}(A \cap C)=\bar{\mu}_{\infty}(A) \bar{\mu}_{\infty}(C)$. From this the theorem would easily follow, because for any $A \in \sigma$ [recalling that $\bar{\Sigma}_{\infty}=\Sigma(F)$ ] we can find a sequence $\left\{A_{n}\right\}$ for which $A_{n} \in F$ for all $n$ and $\lim _{n \rightarrow \infty} \bar{\mu}_{\infty}\left(A_{n} \Delta A\right)$ $=0$. We then would have

$$
\begin{aligned}
\bar{\mu}_{\infty}(A) & =\bar{\mu}_{\infty}(A \cap A)=\lim _{n \rightarrow \infty} \bar{\mu}_{\infty}\left(A \cap A_{n}\right) \\
& =\lim _{n \rightarrow \infty} \bar{\mu}_{\infty}(A) \bar{\mu}_{\infty}\left(A_{n}\right)=\left[\bar{\mu}_{\infty}(A)\right]^{2},
\end{aligned}
$$

so that we would have $\bar{\mu}_{\infty}(A)=0$ or $\bar{\mu}_{\infty}(A)=1$.

We now use a) to obtain the structure of the $\sigma^{n}$. Recall that $\gamma_{0}$ partitions $\bar{B}_{\infty}$ into "vertical" lines. Hence $\bar{T}_{\infty}^{-n} \hat{\gamma}_{0}$ partitions $\bar{B}_{\infty}$ into unions of $2^{n}$ vertical lines in such a way that the image under $\bar{T}_{\infty}^{-n}$ of a line in $B_{0}$ is a set of lines scattered among the $B_{i}$ with a random walk distribution (i.e., the number of lines in $B_{j}$ is $p_{j}^{n} 2^{n}$, with $p_{j}^{n}$ the $n$-step $0 \rightarrow j$ random walk transition probability). We can thus use the central limit theorem to find an $\bar{N}$ such that for any $A \in \Sigma\left(\bar{T}_{\infty}^{-} \bar{N}_{0}\right)$ we have

$$
\mu_{\bar{B}_{\infty}}\left(A \cap \bigcup_{i=-M}^{M} B_{i}\right)<\bar{\varepsilon} \mu_{\bar{B}_{\infty}}(A)
$$

(given $\bar{\varepsilon}$ and $M$ ). Thus given any $\beta \in F$ and any $\varepsilon>0$, we might expect that there would exist an $N$ such that for any $\alpha \in \sigma^{N}=\Sigma\left(\zeta\left[\bar{T}_{\infty}^{-N} \hat{\gamma}_{0}\right]\right)$ we would have

$$
\left|\bar{\mu}_{\infty}(\alpha \cap \beta)-\bar{\mu}_{\infty}(\alpha) \bar{\mu}_{\infty}(\beta)\right|<\varepsilon .
$$

Thus for $\alpha \in \sigma$ we would have $\bar{\mu}_{\infty}(\alpha \cap \beta)=\bar{\mu}(\alpha) \bar{\mu}_{\infty}(\beta)$ for any $\beta \in F$, and the proof would be complete.

The difficulty in the above argument lies in showing that $*$ is valid uniformly as $\alpha$ ranges over $\sigma^{n}$. We bypass this difficulty by using Doob's martingale theorem [20] to directly establish 3). We need the corollary of Doob's theorem which asserts that for a decreasing sequence of $\sum$-algebras, $\Sigma_{n} \downarrow \Sigma_{0}$, and a measurable set $A$ we have

$$
\lim _{n \rightarrow \infty} \mu\left(A \| \Sigma_{n}\right)(x)=\mu\left(A \| \Sigma_{0}\right) \quad \text { a.e. },
$$


where $\mu(. \|$.) denotes conditional measure (with respect to an arbitrary $\sum$-algebra; see [13], Chapter III).

We want to verify that $\sigma_{n} \downarrow v$. Since $\mu(A \| \Sigma)=\mu(A)$ a.e. if and only if $\Sigma=v(\bmod 0)$ we must show that for $A \in \bar{\Sigma}_{\infty}$ we have

$$
\lim _{n \rightarrow \infty} \bar{\mu}_{\infty}\left(A \| \sigma^{n}\right)=\bar{\mu}_{\infty}(A) \quad \text { a.e. }
$$

But, by virtue of the remarks at the beginning of the paragraph before the preceding one, it is not difficult to $\mathrm{see}^{4}$ that $* *$ is satisfied by $A$ of the form $X_{\alpha}^{\left\{n_{j}\right\}}$ [see proof of Theorem IV.2] and hence by all $A \in \bar{\Sigma}_{\infty}$, so the proof is complete.

\section{Ergodic Properties of Infinite Continuous System}

The continuous case can be treated similarly to the discrete case. We will therefore limit ourselves to a few remarks, omitting details.

In the previous section we indicated how the system $\bar{\tau}_{\infty}=\left(\bar{X}_{\infty}, \bar{\mu}_{\infty}, \bar{S}_{\infty}\right)$, can be obtained by a Poisson construction from the non-normalizable, one-particle system $\left(\bar{B}_{\infty}, \varrho \mu_{\bar{B}_{\infty}}, \bar{T}_{\infty}\right)$. The auxiliary space $\bar{B}_{\infty}$ could be regarded as a product of the baker's square with the discrete space $\mathbb{Z}$. The continuous models $\tau_{v, \varrho}=\left(X_{\infty}, \Sigma_{\infty}, \mu_{v, \varrho}, S_{t}\right)$, where $v$ is an even probability measure on $\mathbb{R}$, the velocity space of the particle, absolutely continuous at the origin, are flows which can be obtained by a Poisson construction from the auxiliary system $\left(B_{\infty}, \mu_{B_{\infty}}(\varrho, v), T_{t}\right) ; B_{\infty}=B \otimes \mathbb{R}^{2}$ and $d \mu_{B_{\infty}}=d \mu_{B} \otimes \varrho d q \otimes d v$. We have chosen $v$ to be absolutely continuous at the origin so that the probability of finding a particle at rest in any given (finite) interval will vanish.

As already suggested by our notation the only "physically reasonable" invariant probability measures on $\Sigma_{\infty}$ are of the form $\mu_{v, \varrho}, v$ an even probability measure on $\mathbb{R}$. Letting $\hat{\beta}$ denote a Maxwellian measure on $R$ with inverse temperature $\beta$, i.e., $\hat{\beta}(A)=\sqrt{\beta / 2 \pi} \int_{A} e^{-\frac{1}{2} \beta v^{2}} d v, A \subset \mathbb{R}$ (taking the mass of the particles to be unity), we obtain a family of "states" $\left\{\mu_{\hat{\beta}, \varrho}\right\}$ natural from the standpoint of statistical mechanics (since they are infinite volume limits of grand canonical ensembles). The presence here of more general invariant measures corresponding to different velocity distributions is due to the fact that the velocities play a trivial role in the "collisions".

${ }^{4}$ The convergence of $\bar{\mu}_{\infty}\left(A \| \sigma^{n}\right)$ is a tail property, i.e. $\lim _{n \rightarrow \infty} \bar{\mu}_{\infty}\left(A \| \sigma^{n}\right)$, which by Doob's theorem exists a.e., is measurable with respect to $\Sigma_{C}$ for $C$ the complement of any bounded subset of $\bar{B}_{\infty}$. Thus, by the zero-one law for tail events [21], $\lim _{n \rightarrow \infty} \bar{\mu}_{\infty}\left(A \| \sigma^{n}\right)=\bar{\mu}_{\infty}(A \| \sigma)$ is constant, a.e. 
The partition of $X_{\infty}$ which corresponds to the partition $\zeta\left[\gamma_{0}\right]$ has the property that two points $x$ and $x^{\prime} \in X_{\infty}$ belong the same element of this partition if they differ at most by values of some "past" Bernoulli coordinates. In essentially the same way as for $\zeta\left[\gamma_{0}\right]$, this partition is seen to satisfy the conditions by virtue of which it is a $K$-partition. We thus have:

Theorem 6. $\tau_{v, \varrho}$ is a K-flow if $v$ is absolutely continuous at the origin.

\section{Ergodic Properties of a General Class of Infinite Systems}

We are now ready to generalize the results of the last two sections. We will do this by establishing a theorem relating the ergodic properties of a general (base) system $(X, \mu, T)$ to those of $\left(X_{\infty}, \mu_{\infty}, T_{\infty}\right)$, the Poisson system built over $(X, \mu, T)$. The theorem will concern (base) systems which share with $\left(\bar{B}_{\infty}, \mu_{\bar{B}_{\infty}}, \bar{T}_{\infty}\right)$ certain key features. In particular we observe that the group $\mathbb{Z}$ of integers, acting in the natural way upon $\bar{B}_{\infty}$, preserves $\mu_{\bar{B}_{\infty}}$ and commutes with $\bar{T}_{\infty}$. We can thus "reduce" $\left(\bar{B}_{\infty}, \mu_{\bar{B}_{\infty}}, \bar{T}_{\infty}\right)$ to a set $\bar{B}_{n}=\bigcup_{i=-n}^{n} B_{i}$ by replacing $\bar{B}_{\infty}=B \otimes \mathbb{Z}$ by $B \otimes \mathbb{Z}_{2 n+1}\left(\mathbb{Z}_{n}\right.$ denoting the integers considered modulo $n$ ); we obtain in this way (after normalizing the induced measure) the one particle periodic systems $\bar{\tau}(2 n+1)$, which we have shown, Theorem 4.1, to be $K$-systems (in fact, Bernoulli).

Let $X$ have a representation as $\mathbb{R}^{2}$ with $\mu$ defined on Lebesgue sets. (We make this assumption for the sake of convenience of expression; the appropriate generalizations of the definition we give should be clear. We could, in particular, take $(X, \mu)$ to be the product of $\left(\mathbb{R}^{2}, \mu_{\mathbb{R}^{2}}\right)$ with any probability space and proceed in the obvious manner.) Let $T$ be an automorphism of $(X, \mu)$ and let the representation be such that there exist $a, b \in \mathbb{R}$ for which $G_{(a, b)}$, the group generated by $(x, y) \rightarrow(x+a, y)$ and $(x, y) \rightarrow(x, y+b)$, preserves $\mu$ and commutes with $T$. Let $R_{0}=\{(x, y)$ $\left.\in \mathbb{R}^{2} \mid 0 \leqq x<a, 0 \leqq y<b\right\}$ and let us call the translates of $R_{0}$ by the elements of $G_{(a, b)}$ basic rectangles. Let us call rectangles which are unions of basic rectangles compound rectangles. For any compound rectangle $R$ with sides of length $j a$ and $k b$ let $\tau_{R}$ be the dynamical system obtained from $(X, \mu, T)$ by replacing $X$ with $X$ modulo $G_{(j a, k b)}$. We will say that a sequence $R_{i}$ of rectangles converges to infinity if the sequence of lengths of the largest side converges to infinity. $(X, \mu, T)$ will be said to be of periodic K-type if $\tau_{R_{0}}$ has finite entropy ${ }^{5}$ [22], $T\left(R_{0}\right)$ is bounded, and

$(K)$ there exists a sequence $R_{i}$ of compound rectangles converging to infinity such that each of the systems $\tau_{R_{l}}$ is a $K$-system.

5 By virtue of Kouchnirenko's theorem, all classical dynamical systems have finite entropy [1]. 
$(X, \mu, T)$ will be said to be of periodic $M$ type if

$(M)$ there exists a sequence $R_{i}$ of compound rectangles converging to infinity such that each of the systems $\tau_{R_{i}}$ is mixing.

We can now state

Theorem 7.1. If $(X, \mu, T)$ is of periodic K-type (M-type), then $\left(X_{\infty}\right.$, $\mu_{\infty}, T_{\infty}$ ), is a K-system (mixing).

Proof. It follows from $(M)$ that for bounded subsets $A$ and $B$ of $\mathbb{R}^{2}$,

$$
\lim _{n \rightarrow \infty} \mu\left(T^{n} B \cap A\right)=0 \text {. }
$$

The "mixing" assertion then follows from an argument similar to the one given in the proof of Theorem 5.2.

Let $\zeta_{\infty}$ be the partition of $X_{\infty}$ according to the number of particles in each of the fibers of a partition $\zeta$ of $X$. We have seen in Section 5 that $\left(\hat{\gamma}_{0}\right)_{\infty}$ is a $K$-partition for $\bar{\tau}_{\infty}$, where $\hat{\gamma}_{0}$ is the partition of $\bar{B}_{\infty}$ into "vertical" line segments. Letting $P_{\infty}$ be the partition of $\bar{B}_{\infty}$ whose elements are the $B_{i}(i=0,1,-1, \ldots)$, we recall that $\bar{B}_{\infty}$ can be identified with the set of possible $P_{\infty}$-names (what we have previously called "spatial" trajectories) [10], and that $\hat{\gamma}_{0}$ can be identified with the partition of $\bar{B}_{\infty}$ according to "future" $P_{\infty}$-names $\left(\zeta\left(\bigvee_{j=0}^{\infty} T_{\infty}^{-j} P_{\infty}\right)\right)$. We further recall that a key element in the proof of Theorem 5.3 was the observation that by virtue of the central limit theorem the fibers of $\hat{\gamma}_{0}$ expand toward infinity; i.e., the fiber of $\bar{T}_{\infty}^{-n} \hat{\gamma}_{0}$ containing a (fixed) point $x \in \bar{B}_{\infty}$ grows (monotonically) with $n$ in such a way that the fraction of the fiber intersecting any fixed bounded region $A \subset \bar{B}_{\infty}$ approaches zero.

For the problem at hand we proceed similarly. We let $Q_{0}$ be the partition of $X$ into basic rectangles and let $Q$ be a finite partition of $R_{0}$ which is a generator for $\tau_{R_{0}}$. (Since $\tau_{R_{0}}$ has finite entropy, Krieger's theorem guarantees the existence of such a partition [23].) We then obtain $Q_{\infty}$ by forming the "product" of $Q_{0}$ and $Q$ : the atoms of $Q_{\infty}$ are obtained by decomposing each atom of $Q_{0}$ according to $Q$. Finally we let our base-K-partition $\zeta$ be the partition of $X$ according to future $Q_{\infty}$-names. Now the proof that $\zeta_{\infty}$ is a $K$-partition for $\left(X_{\infty}, \mu_{\infty}, T_{\infty}\right)$ is similar to the proof of Theorem 5.3. We need mention only that since, by virtue of $(K)$ and the boundedness of $T\left(R_{0}\right)$, the restriction of $\zeta$ to any of the rectangles $R_{i}$ is a $K$-partition for $\tau_{R_{i}}$, finite partitions of $K$ systems having trivial tails [24], the martingale convergence theorem applied to the $\tau_{R_{l}}$ implies that the fibers of $\zeta$ expand toward infinity ${ }^{6}$, permitting us to infer that the analogue of $* *$ (see proof of Theorem 5.3) is valid for $\left(X_{\infty}, \mu_{\infty}, T_{\infty}\right)$.

${ }^{6}$ To define precisely the concept of expansion toward infinity, we use the canonical systems of measures possessed by the $T^{-n} \zeta[15]$. 
We conclude by using Theorem 7.1 to prove that a Lorentz gas [25] forms a $K$-system. Sinai has shown that (apart from possible pathological situations) the motion of a particle in a two dimensional rectangle, with periodic boundary conditions, containing convex barriers from which the particle, which otherwise moves freely, undergoes elastic collisions induces a $K$-flow on the unit tangent bundle of the rectangle [2]. Thus the dynamical system representing the motion of a particle in a two dimensional (nonpathological) periodic array of convex barriers (at unit velocity) is of periodic $K$-type. Hence, the Lorentz gas, which is an infinite gas of such particles, all moving with the same speed, with a Poisson configurational measure on regions unoccupied by barriers, is a $K$-system for any discrete time evolution.

The thermodynamic limit of a grand canonical ensemble, which corresponds to having a Maxwellian velocity distribution, is not built over a system of periodic $K$-type (since the speed of a particle is a constant of the motion). We can however use an argument similar to the one given above to conclude that it, too, is a $K$-system; we choose as our base- $K$-partition $\zeta$ the union of the partitions $\zeta(s)\left(s \in \mathbb{R}^{+}\right)$, the base- $K$ partitions on the surfaces of constant speed, and use the fact that such surfaces "support" systems of periodic- $K$-type. (Here we are ignoring the technical problem of showing that the partitions $\zeta(s)$ can be chosen in such a way that their union is a measurable partition. We also observe that although we have shown that our Lorentz gas is a $K$-system under any discrete time evolution, we have not shown it to be a $K$-flow, though an approach similar to the above could probably be exploited to establish this result as well.)

\section{Remarks}

We observe that though the infinite ideal gas and the infinite Poisson systems $\tau_{\infty}$ built on base systems which are of periodic $K$-type are both $K$-systems, there is certainly a sense in which the "mixing" which occurs in $\tau_{\infty}$ is of a less trivial nature than that which occurs in the ideal gas. (Recall that we require that certain finite submodels of $\tau_{\infty}$ be $K$-systems.) This difference is perhaps reflected in differences in the $K$-partitions for the respective systems. Two points, $x$ and $x^{\prime}$, in the phase space of the ideal gas belong to the same element of its $K$-partition if the points $\tilde{x}$ and $\tilde{x}^{\prime}$ obtained from them by deleting all particles outside some region of the auxiliary space coincide, suggesting, perhaps, the "nonlocal" nature of the dissipation of distrubances ${ }^{7}$. Two points, $y$ and $y^{\prime}$, of $X_{\infty}$ belong

7 The infinite volume ideal gas can be obtained by a Poisson construction from the system $\left(\mathbb{R}^{d} \otimes \mathbb{R}^{d},(\beta / 2 \pi)^{d / 2} \exp \left(-\frac{1}{2} \beta \boldsymbol{v}^{2}\right) \boldsymbol{d} \boldsymbol{q} \boldsymbol{d} \boldsymbol{v}, T_{t}^{I}\right)$, where $T_{t}^{I}(\boldsymbol{q}, \boldsymbol{v})=(\boldsymbol{q}+\boldsymbol{v} t, \boldsymbol{v})$. Let $A=\{(\boldsymbol{q}, \boldsymbol{v})$ $\in \mathbb{R}^{d} \otimes \mathbb{R}^{d}: q_{1}+v_{1} t=0$ for some $\left.t \geqq 0\right\}$. One easily verifies, using, e.g., the zero-one law for tail events [21], that $\zeta\left(\Sigma_{A}\right)$ is a $K$-partition for $T_{t}^{I}$. 
to the same element of its $K$-partition if the points $\tilde{y}$ and $\tilde{y}^{\prime}$ obtained from them by factoring out some of the structure of the space $X$ coincide, suggesting, perhaps, a "local" mechanism for the dissipation of disturbances.

This difference can be made more precise by considering in addition to the time evolution also the action of space translations. The ergodic properties of the system under the action of this spacetime group are very different for the ideal gas and the systems considered here, the latter being much more strongly ergodic. This will be considered in a separate publication [26].

We conclude this section by showing that our obtaining a $K$-partition of a very different nature from that of the ideal gas was unavoidable for the model systems considered in Sections 5 and 6. Denoting the one-particle (unit time evolution) ideal gas system by $\left(X^{I}, T^{I}\right)$, we observe that from its $K$-partition we can easily construct a partition $F=\left\{F_{i}\right\}$ of $X^{I}$ such that $T^{I} F_{j}=F_{j+1}$. It is easy to see that the existence of such a partition of the auxiliary space of a Poisson system implies that the system is isomorphic to a Bernoulli shift ${ }^{8}$. We will show that though the systems we have considered may be Bernoulli, they are not of the above type.

Theorem 8.1. Let $T$ be an automorphism of the measure space $(X, \mu)$. If there exists a set $A$ of finite positive measure almost all points of which return to it infinitely often, then we cannot partition $X$ into $\left\{C_{i}\right\}$ in such a way that $T C_{j}=C_{j+1}$.

Proof. Assume we have such an $A$ and $\left\{C_{i}\right\}$ for which $\mu\left(A \cap C_{0}\right)>0$. Let $R_{n}$ denote the set of elements $x \in A$ for which $T^{n} x \in A$. Then the $T^{n}\left(A \cap C_{0} \cap R_{n}\right) \subset A \cap C_{n}$ are disjoint so that

$$
\sum_{n} \mu\left(T^{n}\left(A \cap C_{0} \cap R_{n}\right)\right)=\sum_{n} \mu\left(A \cap C_{0} \cap R_{n}\right)<\mu(A) .
$$

But

$$
\sum_{n} \mu\left(A \cap C_{0} \cap R_{n}\right)=\int_{X} d \mu \sum_{n} \chi\left[A \cap C_{0} \cap R_{n}\right](z)=\int_{A \cap C_{0}} d \mu R(z)=\infty,
$$

where $R(z)$ is the number of integers $n$ for which $T^{n} z \in A$, and $\chi[D]$ is the characteristic function of $D \subset X^{9}$.

Since in a symmetric random walk in one and two dimensions a particle will with probability one return to its original position infinitely often [27], the above theorem applies to the auxiliary space of the models we have considered in Sections 5 and 6 . (For $\bar{\tau}_{\infty}$ we can set $A=B_{0}$.)

\footnotetext{
$8 \quad \Sigma_{F_{0}}$ is an independent generator for $T^{I}$, since $T^{I} \Sigma_{F_{J}}=\Sigma_{F_{J}+1}$.

9 This argument is due to Oscar Lanford.
} 
We observe that all that is required for the above argument is that the measure of the subset of $A$ whose points return to $A$ infinitely often be nonvanishing. If this is not the case we will say that $A$ is nonrecurrent. Strengthened in this way, the theorem admits of a partial inverse.

Theorem 8.2. Let $\mu$ be totally $\sigma$-finite. If all sets of finite measure are nonrecurrent there exists a partition $\left\{C_{i}\right\}$ of $X$ for which $T C_{k}=C_{k+1}$. (Hence the Poisson system built over $(X, \mu, T)$ is isomorphic to a Bernoulli shift.)

Proof. We have $X=\bigcup_{n} D_{n}$, with $D_{n} \subset D_{n+1}$ and $\mu\left(D_{n}\right)<\infty$ for all $n$, for some sequence $\left\{D_{n}\right\}, n \geqq 1$. Let $E_{n}$ be the set of points which will eventually be in $D_{n}$. Let $F_{1}=E_{1}$ and $F_{n}=E_{n}-E_{n-1}, n>1$. Let $f$ be the measurable function from $X$ to $\mathbb{Z}$ such that for $x \in F_{k}, f(x)$ is the largest integer $n$ for which $T^{n}(x) \in D_{k} . f$ is defined almost everywhere, and $f(T x)=f(x)-1$. We therefore obtain a partition $\left\{C_{i}\right\}$ satisfying $T C_{i}=C_{i+1}$ by setting $C_{j}=\{x \mid f(x)=-j\}$.

Since all the states of a random walk in more than 2 dimensions are transient [27], the above theorem is easily seen to apply to the (auxiliary) space representing such a random walk. Furthermore, the analog of the $K$-partition of $\bar{\tau}_{\infty}$ is easily seen to be a $K$-partition for the Poisson system built over a random walk (path) space in any finite number of dimensions (with infinite stationary measure) ${ }^{10}$. Thus a random walk in more than 2 dimensions provides a basis for a system in which a $K$-partition of the ideal gas type and a $K$-partition of the $\bar{\tau}_{\infty}$ type are present simultaneously.

These remarks apply as well to the higher dimensional generalizations of the continuous system $\tau_{v, \varrho}$ considered in Section 6; the two dimensional generalization of the periodic field of barriers could be taken, say, to be a square grid from which particles are either reflected or transmitted according to the same rules as in the one dimensional case.

Acknowledgement. We wish to thank Michael Aizenman and Oscar E. Lanford III for very valuable discussions.

\section{References}

1. Arnold, V.I., Avez, A.: Ergodic problems of classical mechanics. New York: Benjamin 1968

2. Sinai, Y.G.: Russ. Math. Surveys 25, 137 (1970)

3. Volkovysskii, K. L., Sinai, Y. G.: Funkts. Analiz 5, No. 3, 19 (1971)

4. Sinai, Y.G.: Funkts. Analiz 6, No. 1, 41 (1972)

5. Pazzis, O., de: Commun. math. Phys. 22, 121 (1971)

6. Aizenman, M., Goldstein,S., Lebowitz,J.L.: (To appear)

7. Landford III, O.E., Lebowitz, J.L. : (To appear)

\footnotetext{
10 This system is simply a higher dimensional generalization of $\bar{\tau}_{\infty}$.
} 
8. Landford III,O.E.: Contribution in Boltzman equations (Theory and applications) proceedings symposium Vienna, Sept. 1972. Berlin-Heidelberg-New York: Springer 1973

9. Arnold,V.I., Avez,A.: Ergodic Problems of Classical Mechanics, pp. 7-9, 125, 126 New York: Benjamin 1968

10. Shields,P.: The theory of Bernoulli shifts. University of Chicago Press 1973

11. Ruelle, D.: Statistical mechanics. Rigorous results. New York: Benjamin 1967

12. Adler,R.L., Shields,P.C.: Skew products of Bernoulli shifts with rotations. Israel J. Math. (to appear)

13. Billingsley,P.: Ergodic theory and information, p. 33. New York: Wiley 1965

14. Feller, W.: An introduction to probability theory and its applications, Vol 1, p. 393. New York: Wiley 1968

15. Rohlin,V.A.: Am. Math. Soc. Transl. (1) 10, 1-54 (1962)

16. Parry, W.: Entropy and generators in ergodic theory, pp.34-38. New York: Benjamin 1969

17. Kolmogorov, A. N.: Dokl. Akad. Nauk SSSR 119, No. 5, 861-864 (1958)

18. Rohlin, V.A.: Am. Math. Soc. Transl. (2) 49, 171-240 (1966)

19. Rohlin, V.A.: Am. Math. Soc. Transl. (1) 10, pp. 24, 37 (1962)

20. Doob, J.L.: Stochastic processes, VII, 4. New York: Wiley 1953

21. Feller, W.: An introduction to probability theory and its applications, Vol. 2. New York: Wiley 1971

22. Billingsley,P.: Ergodic theory and information. Chapter 2. New York: Wiley 1965

23. Smorodinsky, M.: Ergodic theory, entropy, Theorem 9.7, p. 56. Lecture Notes 214. Berlin-Heidelberg-New York: Springer 1970

24. Smorodinsky, M.: Ergodic theory, entropy. Theorem 7.9, p. 38, Lecture Notes 214. Berlin-Heidelberg-New York: Springer 1970

25. Gallavotti, G.: Phys. Rev. 185, 308 (1969)

26. Goldstein, S.: (To appear)

27. Feller, W.: An introduction to probability theory and its applications, Vol. 1, pp. 360-361. New York: Wiley 1968

Communicated by G. Gallavotti

J. L. Lebowitz

Belfer Graduate School of Science

Yeshiva University

New York, N. Y. 10033, USA 